Service social

\title{
"Profil de la recherche en gérontologie au Québec ", numéro spécial de Le Gérontophile, vol. 6, no 3, 1984
}

\section{Mohammed Khalid}

Volume 34, numéro 1, 1985

Personnes âgées, milieux de vie et pratiques sociales

URI : https://id.erudit.org/iderudit/706259ar

DOI : https://doi.org/10.7202/706259ar

Aller au sommaire du numéro

Éditeur(s)

École de service social de l'Université Laval

ISSN

1708-1734 (numérique)

Découvrir la revue

Citer ce compte rendu

Khalid, M. (1985). Compte rendu de ["Profil de la recherche en gérontologie au Québec ", numéro spécial de Le Gérontophile, vol. 6, no 3, 1984]. Service social, 34(1), 182-183. https://doi.org/10.7202/706259ar d'utilisation que vous pouvez consulter en ligne.

https://apropos.erudit.org/fr/usagers/politique-dutilisation/ 


\section{RECENSIONS}

"Profil de la recherche en gérontologie au Québec", numéro spécial de Le Gérontophile, vol. 6, no 3, 1984.

Sous la présidence de Nicolas Zay, le Comité de recherche de l'Association québécoise de gérontologie fait montre d'un dynamisme tout à fait remarquable. Nous lui devons ce "Profil de la recherche en gérontologie au Québec". À plus d'un titre, la lecture de ce très important document en laissera plusieurs songeurs: il questionne, en tout cas, plus qu'il ne console, et l'on saura gré au Comité d'obliger les chercheurs à une lucide prise de conscience des orientations et caractéristiques de leurs travaux.

Un questionnaire postal (reproduit en annexe du numéro) a permis la collecte d'informations sur les recherches québécoises en gérontologie pour la période allant de 1977 à 1982. L'analyse des données obtenues a été effectuée par traitement informatique et vingt-six tableaux viennent graphiquement émailler un texte qu'on découvre, avec surprise, d'une lecture bien plus agréable qu'il n'est habituel dans un rapport de ce genre.

On se penche, d'abord, sur les caractéristiques des 128 projets de recherche recensés. On constate, d'emblée, qu'en gérontologie, la recherche en est encore à ses débuts. Ceci est confirmé par le fait que 61 de ces études se réclament de l'étiquette "descriptive" ou "exploratoire", et par la grande variété des thèmes abordés qui ne laisse déceler aucune orientation nette.

Ces thèmes paraissent choisis en fonction des activités, prévues ou en cours, des organismes auxquels les chercheurs sont rattachés : une situation qui profite peut-être davantage à ces organismes qu'à la recherche en gérontologie elle-même.

Le document montre bien, en tout cas, la très grande difficulté à trouver un financement décent (pour ne pas dire désintéressé): le quart des projets comptant sur des montants inférieurs à $5000 \$$, et les deux tiers sur moins de $30000 \$$. Même si on travaille consciencieusement, on se retrouvera vite limité avec si peu. Les bailleurs de fonds veulent sans doute des résultats concrets et immédiats; ils achèteraient plus volontiers une réponse à leurs problèmes qu'ils ne seraient prêts à commanditer une recherche dont l'utilité, pour eux, ne paraîtrait qu'à plus long terme : ce n'est pas dit... mais on peut le penser! Le Comité, quant à lui, parle d'un "saupoudrage" qui n'a rien d'un sérieux soutien stratégique à la recherche.

Une autre constatation peut sembler des plus alarmantes. Une section importante de ce document établit un profil des équipes de recherche. On y découvre que la majorité des chercheurs n'ont pas de poste permanent dans l'organisme d'attache responsable de la gestion de leur recherche. Dans ce 
manque de permanence, on pourrait voir une explication à la vulnérabilité des équipes de recherche sur le plan technique et, surtout, à l'absence de continuité dans le développement d'un plan d'ensemble. C'est peut-être là, à nouveau, poser différemment le crucial problème du financement.

Conscients de la rareté des ressources, certains chercheurs peuvent souhaiter une concentration sur certains aspects stratégiques de l'extension des connaissances. Le Comité fait sienne cette recommandation et propose certains axes de recherche à privilégier :

"Le programme de recherche que l'A.Q.G. aurait intérêt à promouvoir devrait être axé sur les aspects sociaux, économiques et médicaux du vieillissement, et cela de façon à ce qu'on puisse utiliser les ressources disponibles aussi efficacement que possible, améliorer les mesures d'ordre social et sanitaire, y compris la prévention du déclin fonctionnel, des incapacités, des maladies et de la pauvreté dues à l'âge ainsi que la coordination des soins et des services sociaux destinés aux personnes âgées. " (P. 19.)

C'est dire beaucoup; c'est le dire bien. La gérontologie, du reste, sera peut-être jugée socialement rentable et justifiée de mobiliser de plus importantes subventions à la recherche si elle privilégie une telle orientation.

Le Comité mentionnera d'autres rôles possibles pour l'A.Q.G., tant auprès des organismes qui financent ou gèrent les recherches qu'auprès des chercheurs eux-mêmes.

Ce document arrive à point. Le Comité s'est mis à l'écoute des chercheurs. Il analyse, il constate, il interroge. Et il propose. On ne peut absolument pas ne pas en tenir compte.

Professeur à l'Université du

Mohammed KHALID

Québec à Hull,

président de l'Association

québécoise de gérontologie.

Les enjeux après 50 ans, par Louis Plamondon, Gilles Plamondon et Jean CARETTE, Paris, Robert Laffont, 1984, 213 pages.

Avec l'espérance de vie après soixante ans qui augmente progressivement, la généralisation des régimes publics et privés de retraite et la diminution, voulue ou non, de la durée de la vie active, se trouvent réunies les conditions qui font de la retraite une nouvelle période de vie. Apanage d'une minorité au début du siècle, elle devient la condition générale de centaines de milliers d'individus. Et si ceux-ci veulent poursuivre leur vie en situation de non-travail d'une manière enrichissante, il y va de leur intérêt de s'y préparer. C'est dans ce cadre qu'il faut examiner le présent ouvrage. Les auteurs se sont fondés sur les 